Requisitos para madera estructural según el CSCR-2010 y su aporte en la investigación

REQUIREMENTS FOR STRUCTURAL WOOD ACCORDING TO THE CSCR-2010 AND ITS INPUT IN RESEARCH

Ing. Guillermo González Beltrán, Ph.D.

Coordinador General de Laboratorios

LanammeUCR, Costa Rica

guillermo.gonzalez@ucr.ac.cr

Índices y Bases de Datos:

\section{latindex ucrindex}

revistas.ucr.ac.cr/index.php/materiales

(c) lanamme.ucr.ac.cr

@ metodosymateriales.lanamme@ucr.ac.cr
Políticas de Uso:

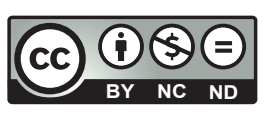

Revista Métodos y Materiales por LanammeUCR se distribuye bajo: Licencia Creative Commons Atribución-NoComercial-SinDerivar 4.0 Internacional. ISSN impreso: 2215-342X. ISSN electrónico: 2215-4558 


\title{
Requisitos para madera estructural según el CSCR-2010 y su aporte en la investigación
}

\author{
REQUIREMENTS FOR STRUCTURAL WOOD ACCORDING TO THE CSCR-2010 AND ITS INPUT IN RESEARCH
}

Ing. Guillermo González Beltrán, Ph.D.

Coordinador General de Laboratorios

LanammeUCR, Costa Rica

guillermo.gonzalez@ucr.ac.cr

Fecha de recepción: 6 de febrero de 2017 / Fecha de aprobación: 8 de junio de 2017

\section{RESUMEN}

Este artículo trata sobre la relación entre las especificaciones del Código Sísmico de Cosa Rica 2010 (CSCR-10) y la investigación generada a partir de ellas. El uso de la madera para construcción en Costa Rica ha venido creciendo desde el año 2000, en el cual se desarrollaron productos industrializados, tales como madera laminada (GLULAM) y madera contrachapada (PLYWOOD). A raíz de esto, el CSCR empezó a trabajar en la elaboración de un capítulo para la versión del año 2010 de ese código (CSCR-10) en donde se desarrolla la especificación para madera estructural. El objetivo de este artículo es destacar el impacto que han tenido los códigos de diseño y las normas de especificación en el desarrollo de la investigación aplicada en el campo del diseño y construcción con madera. Para establecer esta relación, se explican las secciones del CSCR-10 y luego se describen las investigaciones más relevantes. Finalmente, se concluye que el CSCR tiene un gran impacto en el desarrollo de la investigación de la madera estructural en Costa Rica, lo cual se ve reflejado en las investigaciones que se han realizado en un periodo de ocho años.

PALABRAS CLAVE: Madera, madera estructural, conexiones de madera, elementos de madera, marcos de madera, muros de madera, códigos de diseño para madera estructural, normas de especificación para madera.

\section{ABSTRACT}

This paper discusses the relationship between the Costa Rican Seismic Code 2010 (CSCR-10) specifications and the research generated as result of those specifications. The use of timber for construction in Costa Rica has been growing since 2000, including the development of industrialized products such as structural laminated timber (GLULAM) and plywood. Consequently, the CSCR began to work on a chapter for the CSCR-10, in which the specification for structural timber is developed. The objective of this paper is to highlight the impact that the design codes and standard specification have had in the development of applied research in the field of design and construction of timber structures. To establish this relationship, the sections of the CSCR-10 are explained and then, the most relevant investigations are described. Finally, it is concluded that the CSCR has a great impact in the development of research of structural timber in Costa Rica. The previous is reflected on the eight investigations that have been carried out in a period of eight years.

KEYWORDS: Wood, structural wood, wood connections, wooden elements, wood frames, wooden walls, design codes for structural wood, specification standards for wood. 


\section{INTRODUCCIÓN}

El uso de la madera para construcción en Costa Rica ha venido creciendo desde el año 2000, en el cual se desarrollaron productos industrializados tales como madera laminada $y$ madera contrachapada. Aunado a esto, el Código Sísmico de Costa Rica del año 2002 (CFIA, 2003), publica un capítulo dedicado a la madera estructural con el propósito de que fuera extendiéndose en las versiones siguientes. Es por esto que, en el año 2007, se empieza a trabajar en la elaboración de este capítulo para el Código del 2010. Paralelamente, el Instituto de Normas Técnicas de Costa Rica (INTECO) crea un subcomité de maderas para que trabaje en las normas relacionadas con los productos de mayor relevancia. Dado que, según la apreciación del autor, en la comunidad profesional del país, el conocimiento del diseño y la construcción con madera ha disminuido, se torna necesario realizar investigación que respalde las especificaciones y los requerimientos mínimos que se exigen en las normas de los productos y los códigos de diseño. Seguidamente, es necesario implementar un programa de formación técnica y profesional en esa área. El objetivo de este artículo es destacar el impacto que han tenido los códigos de diseño y la norma de especificación en el desarrollo de la investigación aplicada en el campo del diseño y construcción con madera. A continuación, se describe con detalle el antecedente del CSCR-10 y se indica cómo ha influenciado en la investigación. Seguidamente se describen las investigaciones más relevantes, incluyendo las conclusiones más importantes de cada una. Luego se discute sobre esta línea de investigación y finalmente se presentan las conclusiones y recomendaciones.

\section{CAPÍTULO 11 DEL CSCR-2010}

\subsection{Antecedentes}

\subsubsection{Código del Año 2002}

En la versión del CSCR del año 2002, se introdujo el Capítulo 11, dedicado a las especificaciones para estructuras de madera que forman parte del sistema sismorresistente de edificaciones. En esa versión se incluyeron tres seccionesde un par de párrafos cada uno (CFIA, 2003). En la primera sección de "Requisitos Generales" se referenció al "International Building Code" (ICC, 2000) y al "Standard for Load and Resistance Factor Design (LRFD) for Engineered Wood Construction" (AF\&PA/ASCE, 1996), ambos documentos de Estados Unidos. En la segunda sección de "Clasificación Estructural", se describe el sistema tipo marco, limitándolo a un piso de altura y el sistema tipo muro, en el cual se permite hasta cuatro pisos de altura. No se establece un límite en altura en términos de distancia. Se menciona que ambos sistemas son de ductilidad local moderada. En la tercera y última sección de "Requisitos y limitaciones" se establece que para más de dos pisos no se pueden utilizar diafragmas de madera a menos que sean diseñados explícitamente para ese fin. Además, se indican requerimientos constructivos y de diseño para muros de marcos de madera con forro de panel estructural. En los comentarios al CSCR-02 (CFIA, 2007), se explica brevemente el propósito de este capítulo. A pesar de que este primer capítulo es muy básico, genera el precedente para mantener en el CSCR, el tema de la madera estructural y conlleva a que en los años siguientes se desarrolle un nuevo Capítulo 11, más completo, con la revisión e incorporación de los códigos vigentes en el mundo en ese momento.

\subsubsection{Código del Año 2010}

En el año 2007, la Comisión Permanente de Estudio y Revisión del CSCR (CPCSCR), establece un comité, para que desarrolle el capítulo 11 del CSCR-2010. El comité nombrado inicia con una investigación sobre las normas y códigos más recientes, relacionadas con el diseño de estructuras de madera, incluyendo solicitaciones sísmicas y de viento. Debido a la cercanía y a la experiencia de Estados Unidos en este tipo de estructuras, se decide tomar como base la especificación "Special Design Provisions for Wind and Seismic" (AF\&PA, 2005). Esta referencia es la base de la sección 5, que trata el tema de diafragmas y muros. Además, se incluyó para el diseño general de elementos y conexiones, la especificación "National Design Specification for Wood Construction, ASD/ LRFD” (AF\&PA, 2005). Otras referencias importantes para esta primera redacción fueron las normas europeas "Eurocode 5 - Design of Timber Structures" (European Committee for Standardization, 1994), de la cual se tomó la ecuación para estimar la capacidad en cortante de muros y "Eurocode 8 Design Provisions for Earthquake Resistance of Structures" (European Committee for Standardization, 1995), que es la base para definir los sistemas estructurales y sus ductilidades globales asignadas. Un aspecto importante en el desarrollo de este capítulo, fue la definición de los materiales, tales como madera aserrada estructural, madera laminada estructural, madera contrachapada estructural y conectores. A raíz de la ausencia de estos materiales en el mercado costarricense, el Instituto de Normas Técnicas de Costa Rica (INTECO) decide formar un subcomité de maderas, quien se encarga de desarrollar a partir del año 2008, las normas técnicas de la madera aserrada para uso general y estructural. 


\subsection{Estructura del Capítulo 11 del CSCR-2010}

La estructura básica del capítulo 11 del CSCR-2010 está descrita en "Requisitos para madera estructural según el futuro CSCR (González-Beltrán, 2010) y comentada en "Comentarios al Código Sísmico de Costa Rica 2010" (CFIA, 2013). En esta sección se pretende explicar su relación con las investigaciones que se han realizado a raíz de su publicación.

\subsubsection{Factores de Carga y Resistencia}

Después de las generalidades, en donde se especifica el objetivo principal del capítulo, sigue la sección de factores de carga y resistencia. Este capítulo se basa en la especificación NDS 2005 (AF\&PA, 2005). La ecuación básica está dada por:

$$
\lambda \phi_{n} R_{n} \geq R_{u}
$$

Donde el lado izquierdo de la inecuación, representa la resistencia de diseño (capacidad) del elemento o conexión y el lado derecho representa la resistencia requerida (demanda).

El factor $\lambda$, toma en cuenta la duración de la carga en la estructura, por lo tanto, varía con respecto a la combinación de cargas. Por ejemplo, para la combinación de cargas $C U=1.4 C P$ (ecuación 6-1 del CSCR-2010), el factor $\lambda=0.6$. La intención de este factor es limitar el nivel de esfuerzo, de manera que el incremento en la deformación debida al flujo plástico sea estable y no provoque la falla del elemento. En las combinaciones de carga temporal, el factor aumenta, mientras que, en las combinaciones de sismo, el factor es igual a la unidad debido a que no hay efecto de flujo plástico. Es importante indicar que este factor no aplica para los módulos de elasticidad o de cortante. Por ejemplo, para cálculos de deflexiones o cargas críticas de pandeo, donde el esfuerzo no se ve involucrado.

El factor $\phi_{n}$, se conoce como factor de resistencia y es similar al que se utiliza en otros materiales como acero y concreto. Este factor depende del tipo de esfuerzo (flexión, tracción, compresión, cortante, estabilidad y conexiones). Por ejemplo, en el caso de conexiones, el factor es de 0.65 debido a la variabilidad que han presentado los ensayos experimentales.

El término $R_{n}$ representa la resistencia nominal de la sección, zona o conexión que se está analizando. En el NDS 2005 (AF\&PA, 2005), se indica un esfuerzo $F^{\prime}$ que ha sido modificado por ciertos factores de ajuste aplicables entre los cuales se incluyen $\lambda$ y $\phi_{n}$, por lo que el término izquierdo de la Ecuación [1] se determina transformando el valor del esfuerzo ajustado $F^{\prime}$ a un valor de fuerza interna (momento, carga axial, cortante, entre otros). Los valores de ajuste aplicable se encuentran en el NDS 2005 (AF\&PA, 2005) para cada tipo de material. Un factor de ajuste que vale la pena mencionar es el factor de conversión de formato ASD a LRFD, $K_{F}$ Este factor se utiliza para pasar esfuerzos admisibles de diseño a esfuerzos de referencia. Lo anterior se debe a que, en Estados Unidos, la mayoría de las especies están clasificadas en grados estructurales que presentan los valores en esfuerzos admisibles de diseño. Más información sobre la naturaleza de este factor se puede encontrar en la norma de especificación ASTM D 5457-15 (ASTM, 2016).

Finalmente, $R_{u}$ es la resistencia de requerida o carga última, que se determina de acuerdo con los códigos oficiales de cada país y el criterio profesional.

\subsubsection{Materiales}

La sección de Estándares y Materiales, define en cuatro subsecciones, los materiales más utilizados en el diseño y construcción de estructuras de madera. En el mundo actual, es indispensable que los materiales de construcción estén normalizados. Es por esto que, en el 2008, se crea un comité de maderas en el Instituto de Normas Técnicas de Costa Rica (INTECO). El objetivo de este comité, es normalizar los productos de madera comercializados en el país. Hasta el momento, se han finalizado tres normas: Terminología de maderas (INTECO, 2015), Madera aserrada para uso general (INTECO, 2011) y Madera estructural: clasificación en grados estructurales para madera aserrada mediante una evaluación visual (INTECO, 2011). La última es de interés para el CSCR y se basa en la norma ASTM D 255. Además, en esa norma se incluyen los valores para diez especies de plantación, obtenidos mediante investigaciones que se llevaron a cabo en el Instituto Tecnológico de Costa Rica (Moya Roque \& González Beltrán, 2014). Otras normas que se piensan desarrollar en el futuro son las de madera laminada estructural (GLULAM) y madera contrachapada estructural (PLYWOOD). Estos materiales son utilizados en muchos países en donde la madera para construcción tiene un nivel alto de industrialización. En la sección 3.1 de este artículo se presentan las investigaciones realizadas sobre algunas propiedades de los materiales definidos en el CSCR.

\subsubsection{Sistemas Estructurales y Ductilidad}

Esta sección define las ductilidades globales asignadas a varios tipos de sistemas estructurales de madera. En la tabla 4.3 del CSCR-10 se definen las ductilidades globales asignadas a varios tipos de sistemas estructurales como marcos, muros y duales, sin embargo, no se pueden aplicar para madera porque su 
comportamiento estructural difiere de los sistemas tradicionales que son normalmente construidos con acero y con concreto. Es por esto que se define una tabla propia para sistemas estructurales de madera (tabla 11.4 en el capítulo 11 del CSCR-10), en los cuales se incluyen los marcos y los muros. La ductilidad global asignada depende de la ductilidad local y la regularidad del sistema estructural. Además, se limita la altura máxima debido a falta de investigación y experiencia de este tipo de sistema en el país. Por ejemplo, para los marcos tipo 3, los cuales poseen conexiones semirrígidas (tienen rigidez rotacional y traslacional), se permite asignar una ductilidad global de 2.0, siempre y cuando la ductilidad local estática de las conexiones sea de 6.0. Debido a lo anterior, se han hecho investigaciones en conexiones, para determinar su ductilidad local y verificar si pueden cumplir este requisito (ver Sección 3.2 de este artículo).

\subsubsection{Diafragmas y Muros}

En esta sección se definen los requisitos especiales para los diafragmas y muros de madera que se construyen con un marco y se forran con paneles estructurales. Las capacidades nominales se permiten obtener de las tablas incluidas en las provisiones especiales para sismo y viento (AF\&PA, 2005) siempre y cuando los materiales cumplan con la calidad que se establece en ellas. Esto conllevó a realizar las investigaciones que se describen en la Sección 3.3 de este artículo. Con los resultados de estas investigaciones también se revisan las ecuaciones presentadas en el CSCR-10 para determinar la rigidez y deflexión elástica de los muros.

\subsubsection{Conexiones}

Se definen tres tipos de conexión, según su capacidad para transmitir momentos de flexión y su capacidad para deformarse, una vez superado su rango elástico. Las conexiones articuladas se suponen sin capacidad para transmitir momentos de flexión (pueden rotar libremente). Por lo tanto, se asume que solo transmiten fuerzas axiales y cortantes. Las conexiones rígidas pueden transmitir fuerzas de flexión, pero no se pueden deformar más allá del rango elástico (son frágiles). Por último, las conexiones semirrígidas pueden trasmitir momentos de flexión y deformarse más allá del rango elástico, sin que su capacidad disminuya. Las últimas son las de mayor interés para el diseño de estructuras sismorresistentes, pues pueden disipar energía mediante deformación. Las investigaciones de la sección 3.2 y 3.3, estudian el comportamiento de este tipo de conexiones entre elementos de marcos rígidos (viga - columna) y entre paneles estructurales y marcos respectivamente.

\subsubsection{Calidad e Inspección}

El tema de control de calidad e inspección está relacionado con las especificaciones técnicas que se indiquen en los planos constructivos. Es por esto, que en el CSCR se indica la información mínima que deben contener los planos constructivos, de manera que sea verificable durante el control de calidad e inspección. Ver más detalle en los comentarios del CSCR-10 (CFIA, 2013).

\section{INVESTIGACIONES REALIZADAS}

En esta sección se presentan las investigaciones que se han realizado a raíz de la publicación del capítulo 11 del CSCR-2010.

\subsection{Materiales}

\subsubsection{Aplastamiento y Esfuerzo de Flexión}

En el diseño de conexiones de madera, se utiliza el esfuerzo de aplastamiento entre el conector y la madera $\left(F_{e}\right)$ y el esfuerzo de flexión del conector $\left(F_{Y b}\right)$. Con estos dos parámetros y la geometría de la conexión, se pueden calcular los distintos modos de falla para carga lateral (cortante). En la especificación NDS 2005 (AF\&PA, 2005), se permite utilizar ecuaciones empíricas para el cálculo de $F_{e}$ si no se determina experimentalmente. Para conectores con diámetros $(D)$ menores o iguales que $6.4 \mathrm{~mm}, \mathrm{Fe}$ se relaciona con la densidad relativa seca al horno $\left(G_{0}\right)$ y es independiente de la dirección del aplastamiento con respecto al grano, mientras que para diámetros mayores de $6.4 \mathrm{~mm}$, Fe se relaciona únicamente con $G_{0}$ para aplastamiento paralelo al grano y con $G_{0}$ y $D$ para aplastamiento perpendicular al grano $\left(F_{e} \perp\right)$. En el año 2010 se llevó a cabo una investigación (Quesada Solís \& González Beltrán, 2010) en donde se estudiaron las propiedades de aplastamiento y de esfuerzo de flexión para conexiones construidas con maderas y conectores comercializados nacionalmente, variando el tipo y el tamaño de los conectores. Se utilizaron tres especies de madera: teca (Tectona grandis L.f.), melina (Gmelina arborea Roxb) y pino radiata (Pinus radiata) y tres tipos de conectores (clavos, tornillos y pernos). Para cada uno de los conectores se utilizaron 4 diámetros diferentes. La Figura 1 muestra el montaje de los ensayos realizados para este estudio (Quesada Solís \& González Beltrán, 2010). 

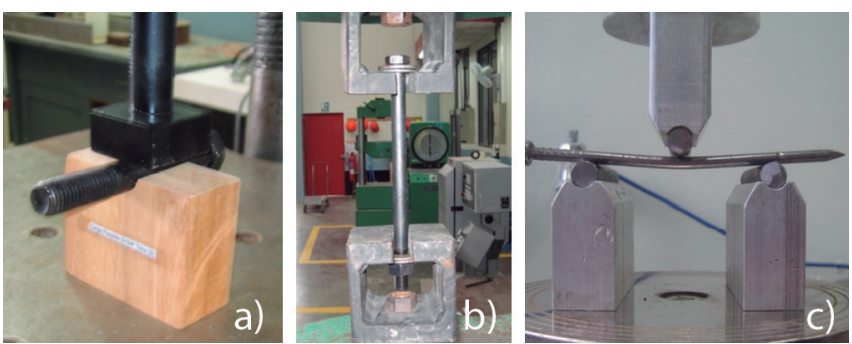

Figura 1. Ensayos realizados para determinar $\mathrm{F}_{\mathrm{e}}$ y $\mathrm{F}_{\mathrm{Yb}}$. (a) Ensayo para determinar $F_{e}$ (b) Ensayo para determinar $F_{y}(c)$ Ensayo para determinar $\mathrm{F}_{\mathrm{Yb}}$. (Quesada Solís \& González Beltrán, 2010).

Las conclusiones de este estudio fueron las siguientes:

- El esfuerzo de aplastamiento paralelo es aproximadamente 1.5 veces el correspondiente esfuerzo de aplastamiento perpendicular.

- El esfuerzo de aplastamiento paralelo y perpendicular depende de la densidad de la madera, pero no se logra demostrar que en algún caso dependa del diámetro.

- Los valores de esfuerzo de fluencia en flexión para los pernos son cercanos a los valores teóricos.

- Los valores de esfuerzo de aplastamiento y flexión en conectores de diámetro pequeño no concuerdan con los valores nominales de la especificación NDS-05 (AF\&PA, 2005), por lo que se recomienda realizar mayor investigación.

\subsubsection{Resistencia de Conexiones}

Además de las propiedades mencionadas en la sección anterior, es recomendable realizar ensayos simples a las conexiones, para verificar que las ecuaciones empíricas y teóricas son confiables para efectos de diseño. En el año 2009, se realizaron este tipo de ensayos en el Laboratorio de Productos Forestales de la Universidad de Costa Rica (Arias, 2010). Estos ensayos se ejecutaron de acuerdo con la norma ASTM D 1761 (ASTM, 2012). Por ejemplo, las cargas de extracción para clavos y tornillos se determinan mediante fórmulas empíricas, que dependen de la longitud de penetración del clavo o tornillo en el elemento principal (el que recibe la punta), el diámetro del conector y la densidad relativa seca al horno de la madera. En la Figura 2(a) se muestra el ensayo de extracción que se aplica a los clavos y tornillos $\left(Z_{w}\right)$. La Figura 2(b) muestra un ensayo para determinar la carga lateral de una conexión simple con clavos o tornillos (Z). En la Figura 2(c) y Figura 2(d) se muestra el ensayo de carga lateral para una conexión apernada con el elemento principal paralelo $(Z)$ y perpendicular a la carga respectivamente $\left(Z_{\perp}\right)$.
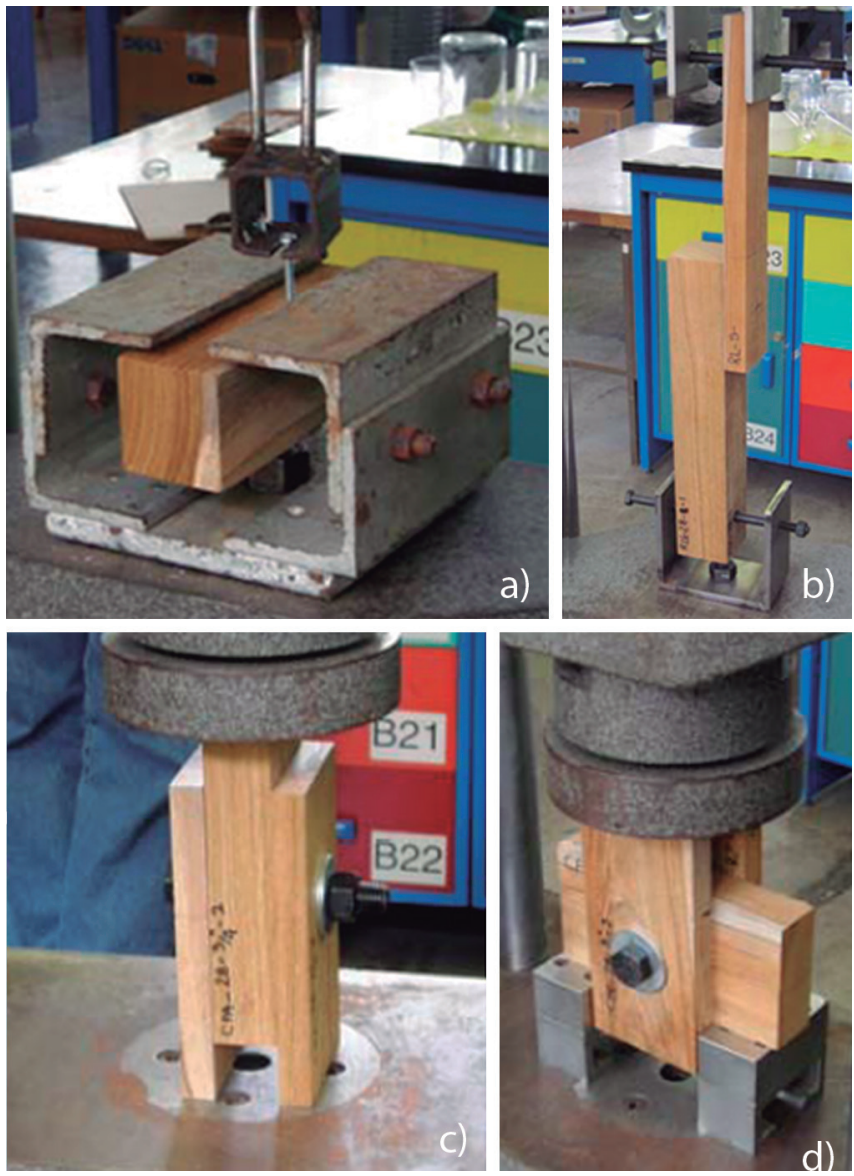

Figura 2. Ensayos realizados para determinar $Z$ y $Z_{w}$ (a) Ensayo para determinar $Z_{w}$ (b) Ensayo para determinar $Z$ en clavos y tornillos (c) Ensayo para determinar $Z$ en pernos (d) Ensayo para determinar $Z_{\perp}$ en pernos. (Arias, 2010).

Para el estudio mencionado, se utilizó únicamente madera de Teca (Tectona grandis L.f.) con 9 y 28 años de edad. Se utilizaron también los siguientes tipos de conectores: pernos de 3 diámetros diferentes, clavos lisos, clavos helicoidales, tornillos para madera y tornillos para metal. Para los clavos y los tornillos se utilizó un único diámetro.

Las conclusiones de este estudio fueron las siguientes:

- La resistencia a la extracción de los tornillos superó 5 veces la de los clavos. Los clavos helicoidales mostraron mayor resistencia que los lisos.

- En resistencia lateral, los tornillos y los clavos helicoidales presentaron valores superiores a los de los clavos lisos, sin embargo, la falla es frágil.

- Los valores teóricos para el cálculo nominal son conservadores, por lo que las ecuaciones teóricas y empíricas son recomendables. 
- No se observó diferencia entre la edad de la madera y la resistencia en cargas de extracción y laterales para clavos y tornillos.

\subsection{Conexiones}

Como se mencionó en la sección 2.3.5, las conexiones entre elementos de madera son indispensables para proveer ductilidad al sistema sismorresistente. A continuación, se describen las principales investigaciones experimentales que se han llevado a cabo en conexiones entre elementos de madera. El objetivo de estos ensayos es determinar la ductilidad local estática que pueden desarrollar estas conexiones utilizando materiales disponibles en el mercado costarricense.

\subsubsection{Conexiones Momento-Resistentes de Configuración Rectangular}

En el año 2009, se llevó a cabo una investigación para determinar el comportamiento y ductilidad de una conexión típica entre una viga y una columna de un marco de madera laminada (Villalobos Ramírez \& González Beltrán, 2009). Se utilizó una configuración rectangular de pernos y placa de acero insertada en la viga y la columna (ver Figura 3a). Los pernos utilizados son ASTM A 325 con un diámetro de 12.7 $\mathrm{mm}$. La viga es una sección de madera laminada de pino radiata (Pinus radiata) de $135 \times 400 \mathrm{~mm}$ y la columna está compuesta por dos secciones de $100 \times 270 \mathrm{~mm}$ del mismo material de la viga. La placa conectora es de acero ASTM A 36 y tiene un espesor de $6.35 \mathrm{~mm}$. Se construyeron tres especímenes, de los cuales, uno se falló con carga monotónica y los otros dos con carga cíclica. Se registra el momento y la rotación de la conexión durante todo el proceso de carga hasta la falla. La Figura 6 muestra el montaje experimental típico. En la Figura 3b se observa el modo de falla típico y en la Figura 3c se muestra la deformación de uno de los pernos y el aplastamiento en los agujeros respectivos.
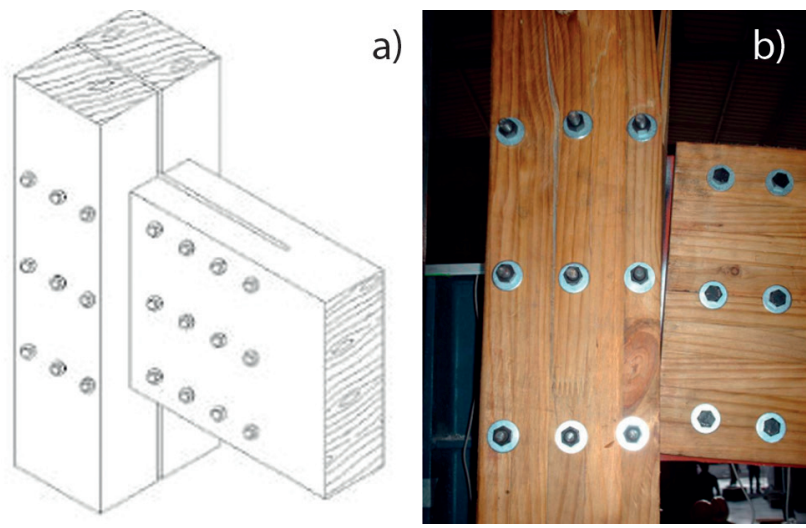


Figura 3. Conexión viga - columna de configuración rectangular. (a) Espécimen (b) Falla en la columna (c) Deformación del perno y aplastamiento en la madera. (Villalobos Ramírez \& González Beltrán, 2009).

Las conclusiones de este estudio fueron las siguientes:

- La ductilidad desarrollada por la conexión varía entre 2.2 y 2.5 , lo cual no es suficiente para desarrollar una ductilidad global de 2.0 en un marco rígido. El CSCR-10 indica que se debe alcanzar una ductilidad estática de 6.0.

- La capacidad cíclica es aproximadamente un $85 \%$ de la capacidad monotónica, lo cual coincide con investigaciones internacionales, aunque hace falta mayor corroboración.

- El momento resistente de diseño es aproximadamente el $80 \%$ del momento experimental en carga cíclica. El momento nominal aproxima bien la resistencia experimental en carga monotónica, pero es mayor que el momento experimental en carga cíclica.

\subsubsection{Conexiones Momento-Resistentes de Configuración Circular}

Continuando con la línea de investigación anterior, en el año 2010 se realizó otra investigación similar, pero utilizando una configuración circular (Chacón Rodríguez, 2010). En este caso, no se utilizó placa conectora y se utilizó doble columna de $50 \times 400 \mathrm{~mm}$ cada una y una viga de $140 \times 400 \mathrm{~mm}$ (Figura 4a). Los pernos utilizados son ASTM A 307 Grado A, con un diámetro de $9.5 \mathrm{~mm}$ y se colocaron en un patrón de tres círculos. Se construyeron seis especímenes en total: tres de pino radiata y tres de melina (Gmelina arborea). De cada grupo, se falló uno en carga monotónica y dos en carga cíclica. Se registra el momento y la rotación de la conexión durante todo el proceso de carga hasta la falla. La Figura 6 
muestra el montaje experimental típico. En la Figura 4b se observa el modo de falla típico y en la Figura 4c se muestra la deformación de dos pernos y el patrón de agrietamiento en la conexión.
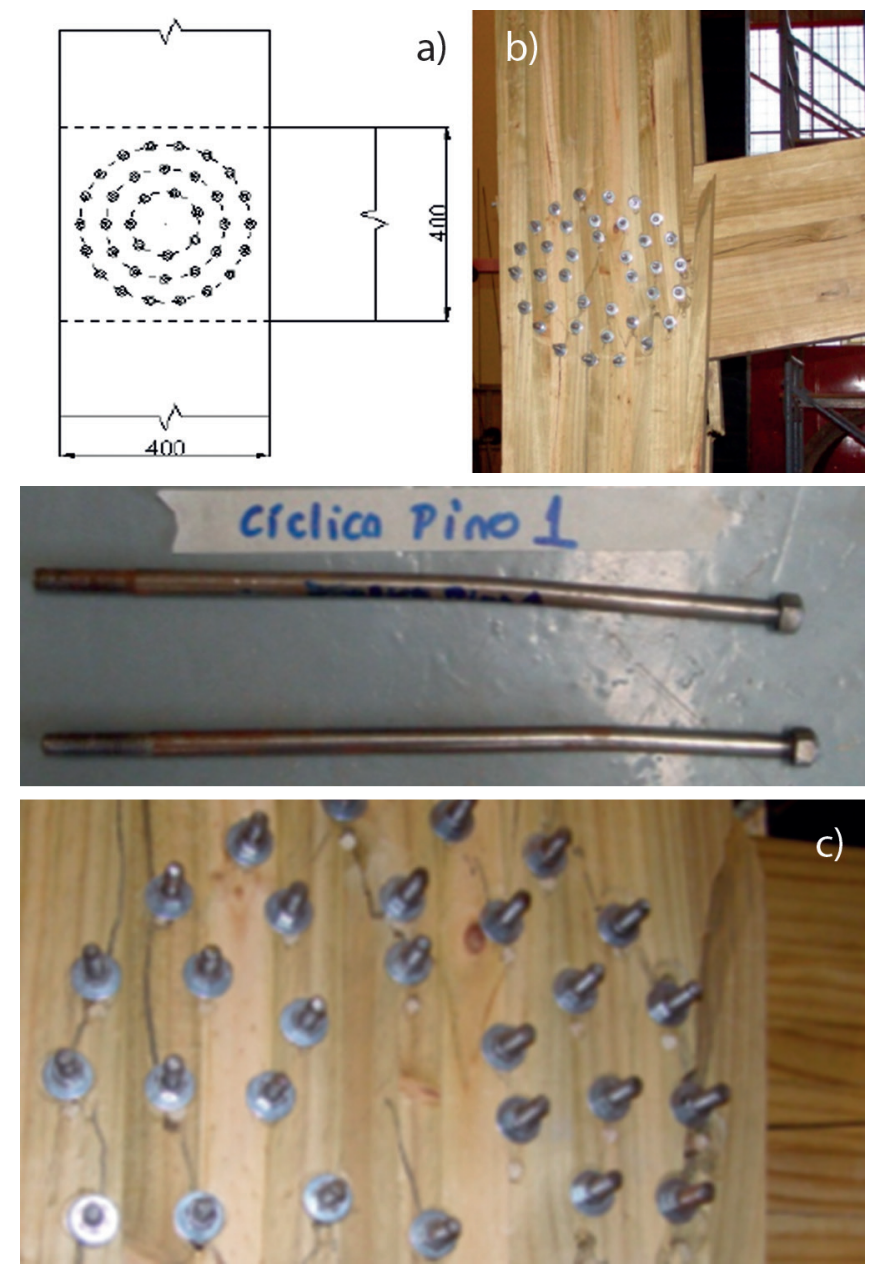

Figura 4. Conexión viga - columna de configuración circular. (a) Espécimen (b) Falla en la conexión y columna (c) Deformación del perno y patrón de agrietamiento en la conexión. (Chacón Rodríguez, 2010).

Las conclusiones de este estudio fueron las siguientes:

- La ductilidad desarrollada por la conexión varió entre 1.4 y 2.88 , lo cual no es suficiente para desarrollar una ductilidad global de 2.0 en un marco rígido.

- La resistencia experimental cíclica fue similar a la resistencia experimental monotónica.

- El momento resistente de diseño estimado es un $56 \%$ del momento experimental en carga cíclica. El momento nominal es menor que el momento experimental un $32 \%$.

\subsubsection{Conexiones Momento-Resistentes de Configuración Circular y Rectangular}

Finalmente, en el año 2013 se realizó una investigación, en la cual se combina la configuración circular (en la viga) con la rectangular (en la columna), con el propósito de darle mayor ductilidad a la conexión y utilizar de manera más eficiente los pernos (González Ureña \& González Beltrán, 2013). En la Figura 5 a se muestra el detalle de la conexión. La conexión fue diseñada para que la falla se diera en la conexión de la viga, que es precisamente donde se varía el diámetro del perno, de manera que, en las zonas de mayor demanda, se colocan los pernos de mayor diámetro. Se construyeron 3 especímenes de pino radiata. Uno se falló en carga monotónica y los otros dos en carga cíclica. Se registra el momento y la rotación de la conexión durante todo el proceso de carga hasta la falla. En la Figura 5b se observa el modo de falla típico, el cual consistió en el pandeo de la placa conectora, debido a la longitud libre que se dejó entre la viga y la columna para evitar aplastamiento prematuro.


Figura 5. Conexión viga - columna de configuración circular y rectangular. (a) Espécimen (b) Falla por flexión de la placa de unión. 
Las conclusiones de esta investigación se resumen a continuación:

- La ductilidad desarrollada por la conexión varió entre 2.3 - 2.9, lo cual no es suficiente para desarrollar una ductilidad global de 2,0 en un marco rígido.

- El momento resistente de diseño es aproximadamente $48 \%$ del momento experimental en carga cíclica. El momento nominal aproxima bien en carga monotónica, pero es mayor que el momento experimental en carga cíclica un 35\%.

- La capacidad de la conexión se ve limitada por el pandeo local de la placa conectora. Por lo tanto, no se logra desarrollar la capacidad de los pernos.

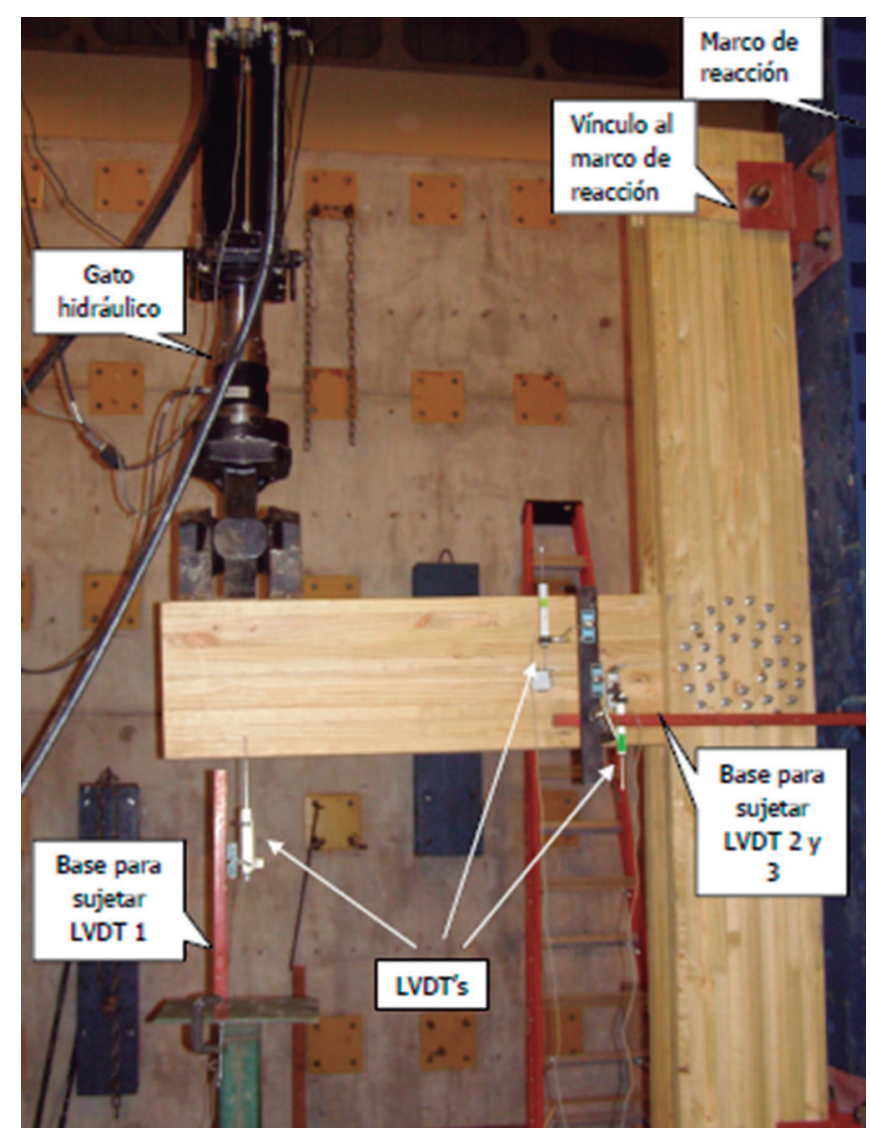

Figura 6. Montaje experimental típico de los ensayos de conexiones en elementos viga - columna.

\subsection{Elementos}

\subsubsection{Muros Sin Anclajes}

El CSCR-10 incluyó un apartado con especificaciones para diafragmas y muros construidos con marco de madera y forro de madera contrachapada estructural (ver sección 2.3.4). Estas especificaciones se basan en el documento de "Provisiones especiales para sismo y viento" (AF\&PA, 2005). En ellas se permite utilizar valores de resistencia y rigidez nominales, para configuraciones típicas en las que se especifica la calidad y geometría de los conectores (clavos), los elementos del marco y del forro. Es por esto que, en el año 2010, se lleva a cabo una investigación, en donde se construyen 6 muros a escala natural con materiales comercialmente disponibles en Costa Rica. Se construyeron tres muros con espaciamiento de clavos de $100 \mathrm{~mm}$ y los tres restantes con un espaciamiento de $150 \mathrm{~mm}$. En ninguno se utilizaron anclajes a la base(Salas Boraschi \& González Beltrán, 2011). De cada grupo de tres muros, uno se falló con carga monotónica y los otros dos con carga cíclica. En la Figura 7 se muestra el montaje experimental utilizado. Durante la prueba se registraron datos de carga y desplazamientos horizontales y verticales. Con la información anterior se obtuvieron valores de resistencia y rigidez en cortante, así como desplazamientos últimos y ductilidades. En la Figura 8 (A1 y A2) se muestra el modo de falla típico, el cual consiste en el levantamiento del poste extremo debido a la deformación excesiva de los clavos.

Las conclusiones principales fueron:

- En todos los casos se logra la plastificación de los conectores más esforzados, los cuales fluyeron en flexión, aspecto que permite al sistema desarrollar ductilidad y evitar una falla frágil.

- Se corrobora que, al no anclar los postes extremos de las paredes, no se alcanza la capacidad teórica dada en la ecuación 11-9 del CSCR-10.

- Deben repetirse los ensayos anclando los postes extremos de las paredes para así determinar si los materiales locales poseen una respuesta adecuada, según los valores estipulados en las tablas de las "Provisiones especiales para sismo y viento" (AF\&PA, 2005). 


\subsubsection{Muros con Anclajes}

Debido a las conclusiones y recomendaciones obtenidas de la investigación anterior, en el año 2013 se lleva a cabo un nuevo estudio similar al anterior(Robles Field \& González Beltrán, 2013), pero en este caso, se utilizan anclajes entre los postes y la base del muro. Los modos de falla típicos se pueden observar en la Figura 8 (B1 a B4). En este caso, las conclusiones fueron las siguientes:

- Las paredes hechas con marco de madera y forro de madera contrachapada, utilizando materiales comercializados en el país, superan la resistencia predicha en los cálculos teóricos, tanto los calculados utilizando la ecuación 11-9 del CSCR 2010 como los de la Tabla 4.3 A del SPDW 2005 (AF\&PA, 2005).

- Es seguro utilizar las metodologías del CSCR-10 para el diseño de muros de corte de madera siempre y cuando los postes se encuentren anclados a la fundación.

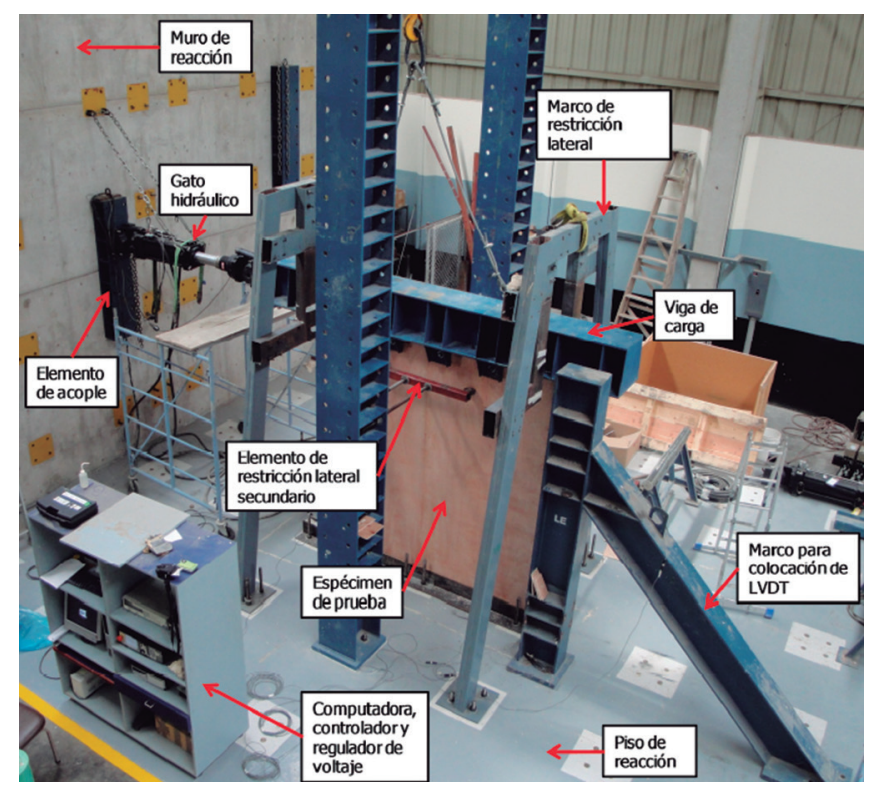

Figura 7. Montaje experimental típico de los ensayos de muros.


Figura 8. Detalle de fallas típicas en los ensayos de muros. A1: levantamiento del poste extremo debido a la deformación de los clavos. A2: clavos deformados.


Figura 8. B1: extracción de clavos entre paneles. B2: falla por tracción del poste extremo. B3: pandeo del panel. B4: deformación de los clavos.

\subsection{Ensamblajes}

En el año 2009, paralelamente a la investigación descrita en 3.2.1, se llevó a cabo otra investigación en donde se probó un marco completo, compuesto por dos columnas y una viga (Figura 9). Éste se cargó con carga horizontal al nivel de la viga y se registró la curva carga vs desplazamiento horizontal del marco al nivel de la viga. Las conexiones viga - columna se diseñaron de manera que alcanzaran el momento nominal antes que las conexiones entre las columnas y la base(Abarca Jiménez \& González Beltrán, 2009).

En este caso, las conclusiones principales son:

- El marco no desarrolla ductilidad pues se comporta linealmente hasta la falla.

- La conexión viga-columna no es capaz de desarrollar una ductilidad local óptima.

- La configuración de la conexión viga-columna en el marco provoca una falla en tracción, perpendicular al grano de la madera que se podría reforzar. 




Figura 9. Montaje experimental de un marco rígido a escala natural.

\section{DISCUSIÓN}

De lo expuesto en las secciones 2 y 3 , se puede deducir que existe una relación estrecha entre el CSCR-10 y la investigación realizada hasta la fecha en el tema de madera estructural. En la Tabla 1 se resumela relaciónentre cada investigación realizada luego de la publicación del CSCR-10 con el apartado correspondiente del capítulo 11 del mismo código.

Analizando la Tabla 1, se puede observar que la investigación ha estado dirigida hacia las propiedades de las conexiones y su ductilidad. Lo anterior se debe a que la madera es un material frágil, que cuando se une con conectores mecánicos de acero dúctil, el sistema puede desarrollar ductilidad y disipar energía mediante la deformación de sus conexiones. Esto es de suma importancia para el diseño de sistemas estructurales sismorresistentes.

Tabla 1. Resumen de las investigaciones relacionadas con el CSCR-10

\begin{tabular}{|c|c|c|}
\hline Sección CSCR-10 & $\begin{array}{c}\text { Investigación } \\
\text { relacionada }\end{array}$ & Resultados \\
\hline $\begin{array}{c}\text { 11.3.5 Conectores } \\
\text { mecánicos } \\
\text { para madera }\end{array}$ & $\begin{array}{c}\text { Ver sección 3.1.1 } \\
\text { y 3.1.2 }\end{array}$ & $\begin{array}{l}\text { Coincidencias y dis- } \\
\text { crepancias entre va- } \\
\text { lores teóricos y valo- } \\
\text { res experimentales }\end{array}$ \\
\hline
\end{tabular}

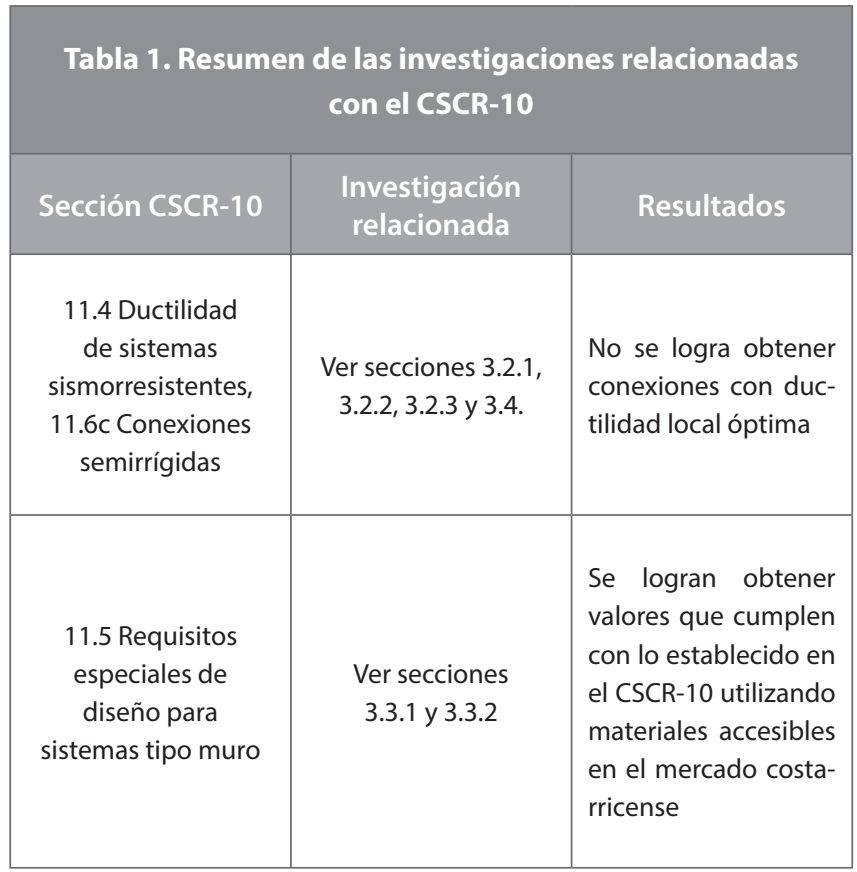

Otro aspecto que es importante destacar, es que los resultados de las investigaciones, algunas veces, conllevan a realizar otra investigación que aclara los puntos que no se pudieron resolver en el estudio anterior. Por ejemplo, en el estudio de los muros sin anclaje de los postes extremos, éstos no alcanzan la resistencia teórica o nominal, mientras que los muros con anclajes sí lograron superar la resistencia nominal establecida en el CSCR-10. Otro caso es el de las conexiones semirrígidas, que en cada una de las investigaciones posteriores se ha tomado en cuenta las experiencias aprendidas en las anteriores. Por ejemplo, la utilización de pernos más dúctiles, de menor diámetro y menor resistencia.

\section{CONCLUSIONES Y RECOMENDACIONES}

Con lo expuesto en este artículo, se concluye que el CSCR tiene un gran impacto en el desarrollo de la investigación de la madera estructural en Costa Rica. Lo anterior se refleja en las investigaciones que se han realizado en un periodo de ocho años, a raíz de la publicación del CSCR-10.

También se puede concluir que el CSCR ha tenido influencia en el desarrollo de normas técnicas de productos relacionados con la madera estructural. Ya se publicó la norma de madera aserrada estructural y actualmente se trabaja en la de madera laminada estructural.

Los resultados de las investigaciones presentadas podrían modificar y complementar ediciones posteriores del CSCR. 


\section{AGRADECIMIENTOS}

Las investigaciones mencionadas en este artículo fueron posibles, gracias al patrocinio de la Comisión Permanente de Estudio y Revisión del CSCR y del Laboratorio Nacional de Materiales y Modelos Estructurales de la Universidad de Costa Rica (LanammeUCR).

El autor agradece a todos los investigadores mencionados y al apoyo del personal técnico por todo el trabajo realizado.

\section{REFERENCIAS}

Abarca Jiménez, A., \& González Beltrán, G. (2009). Comportamiento estructural de marcos de madera laminada con uniones momento-resistentes (ductilidad golbal). X Seminario de Ingeniería Estructural y Sísmica. San José, Costa Rica.

AF\&PA. (2005). National Design Specification for Wood Construction and Supplement. Washington D.C.: AF\&PA.

AF\&PA. (2005). Special Design Provisions for Wind and Seismic with Commentary (2005 ed.). Washington, DC, United States of America: AF\&PA, Inc.

AF\&PA/ASCE. (1996). Standard for Load and Resistence Factor Design (LRFD) for Engineered Wood Construction. United States: American Society of Civil Engineers.

- Arias, M. (2010). Evaluación de la resistencia de diferentes tipos de uniones en madera de Teca. San José, Costa Rica: Universidad de Costa Rica.

American Society for Testing and Materials. (2012). ASTM D1761. Standard Test Methods for Mechanical Fasteners in Wood. Anual Book of ASTM Standards. Volume: 4.10 (pág 10). West Conshohocken: ASTM International.

- American Society for Testing and Materials. (2016). Standard Specification for Computing Reference Resistance of Wood-Based Materials and Structural Connections for Load and Resistance Factor Design. En ASTM, Annual Book of ASTM Standards, Vol 4.10 (pág. 10). West Conshohocken, PA: ASTM International.

Colegio Federado de Ingenieros y Arquitectos (CFIA). (2003). Código Sísmico de Costa Rica 2002. Cartago, Costa Rica: Editorial Tecnológica de Costa Rica.

- Colegio Federado de Ingenieros y Arquitectos (CFIA). (2007). Comentarios al Código Sísmico de Costa Rica 2002. Cartago, Costa Rica: Editorial Tecnológica de Costa Rica.

Colegio Federado de Ingenieros y Arquitectos (CFIA). (2011). Código Sísmico de Costa Rica 2010 (Cuarta ed.). Cartago, Costa Rica: Editorial Tecnológica de Costa Rica.
Colegio Federado de Ingenieros y Arquitectos. (CFIA). (2013). Comentarios al Código Sísmico de Costa Rica 2010. Cartago, Costa Rica: Editorial Tecnológica de Costa Rica.

Chacón Rodríguez, M. J. (2010). Comportamiento estructural de conexiones con configuración circular en elementos de madera en sistemas viga-columna. San José, Costa Rica: Universidad de Costa Rica.

European Committee for Standardization. (1994). Eurocode 5 Design of Timber Structures. Brussels: CEN.

European Committee for Standardization. (1995). Eurocode 8 - Design Provisions for Earthquake Resistance of Structures. Brussels: CEN.

González Ureña, A., \& González Beltrán, G. (2013). Comportamiento estructural de conexiones semirrígidas en marcos sismorresistentes de madera laminada. Congreso de Estructuras 2013, XII Seminario de Ingeniería Estructural y Sísmica (pág. 12). San José, Costa Rica.

González-Beltrán, G. (2010). Requisitos para madera estructural según el futuro CSCR - Investigaciones recientes y normalización. Congreso CIC-2010, (pág. 10). San José, Costa Rica.

ICC. (2000). International Building Code 2000. Illinois: International Code Council, INC.

Instituto de Normas Técnicas de Costa Rica. (2011). INTE 0607-02 Madera aserrada para uso general. Requisitos. San José, Costa Rica: INTECO.

Instituto de Normas Técnicas de Costa Rica. (2011). Madera Estructural - Clasificación en grados estructurales para la madera aserrada mediante una evaluación visual. San José, Costa Rica: INTECO.

Instituto de Normas Técnicas de Costa Rica. (2015). INTE 0607-01 Terminología de maderas. San José, Costa Rica: INTECO.

Moya Roque, R., \& González Beltrán, G. (2014). Esfuerzos admisibles de diseño por grado estructural para nueve maderas de plantación de Costa Rica. Revista Forestal Mesoamericana Kurú, 11.

Quesada Solís, J., \& González Beltrán, G. (2010). Caracterización de propiedades de aplastamiento y esfuerzo de flexión, necesarias para el diseño de conexiones de elementos de madera con materiales existentes en el mercado costarricense. Congreso de Estructuras 2011 y XI Seminario de Ingeniería Estructural y Sísmica. San José, Costa Rica.

Robles Field, A., \& González Beltrán, G. (2013). Comportamiento estructural de paredes livianas con marco de madera y forro de madera contrachapada ante carga horizontal, con anclajes en la base. Congreso Estructuras 2013. XII Seminario de Ingeniería Estructural y Sísmica. San José, Costa Rica. 
Salas Boraschi, B., \& González Beltrán, G. (2011). Comportamiento estructural de paredes livianas con marco de madera $y$ forro de madera contrachapada ante carga horizontal. XI Seminario de Ingeniería Estructural y Sísmica. San José, Costa Rica.

Villalobos Ramírez, F., \& González Beltrán, G. (2009). Comportamiento estructural de conexiones sismorresistentes de elementos de madera laminada en sistemas viga-columna (ductilidad local). X Seminario de Ingeniería Estructural y Sísmica. San José, Costa Rica. 